\title{
The safety and effectiveness of low field intraoperative MRI guidance in frameless stereotactic biopsies of brain tumours-design and interim analysis of a prospective randomized trial
}

\author{
M. Czyż • P. Tabakow • A. Weiser • B. E. Lechowicz-Glogowska • \\ L. W. Zub $\cdot$ W. Jarmundowicz
}

Received: 21 November 2012 / Revised: 2 February 2013 / Accepted: 17 March 2013 / Published online: 3 July 2013

(C) The Author(s) 2013. This article is published with open access at Springerlink.com

\begin{abstract}
The aim of the study was to assess the safety and effectiveness of stereotactic brain tumour biopsy (STx biopsy) guided by low-field intraoperative magnetic resonance imaging (iMRI) in comparison with its frameless classic analogue based on a prospective randomized trial. A pilot group of 42 brain tumour patients was prospectively randomized into a low-field iMRI group and a control group that underwent a frameless STx biopsy. The primary endpoints of the analysis were postoperative complication rate and diagnostic yield, and the secondary endpoints were length of hospital stay and duration of operation. The iMRI group (21 patients) and the control group (21 patients) did not differ significantly according to demographic and epidemiological data. No major postoperative complications were noted in either group. In addition, no significant differences in the diagnostic yield $(p=1.00)$ and length of hospital stay $(p=0.16)$ were observed. The mean total OR time was $111 \pm 24 \mathrm{~min}$ in iMRI and $78 \pm 29 \mathrm{~min}$ in the control group ( $p=0.0001)$. Usage of iMRI may prolong the time of the procedure but seems to be comparable in safety and effectiveness to the standard frameless STx biopsy.
\end{abstract}

Keywords Intraoperative magnetic resonance $\cdot$ Stereotactic biopsy $\cdot$ Brain tumour $\cdot$ Frameless stereotaxy $\cdot$ Image-guided neurosurgery

\footnotetext{
M. Czyż $(\bowtie) \cdot$ P. Tabakow • A. Weiser $\cdot$ L. W. Zub •

W. Jarmundowicz

Department of Neurosurgery, Wroclaw Medical University,

Ul. Borowska 213, 50-556 Wrocław, Poland

e-mail:mt.czyz@gmail.com

B. E. Lechowicz-Głogowska

Department of Anaesthesiology and Intensive Therapy,

Wroclaw Medical University, Wroclaw, Poland
}

\section{Introduction}

Stereotactic brain biopsy (STx biopsy) offers a relatively straightforward, accurate and safe method of obtaining diagnostic tissue [20]. Frameless computer-based neuronavigation is now widely used in brain tumour surgery. It has many advantages over frame-based techniques - it is less time-consuming, involves much less human resources, it is better tolerated by the patient, more cost-effective and provides similar accuracy to the rigid frame $[8,13]$. One of the methods applied to improve diagnostic yield and safety is the usage of intraoperative magnetic resonance imaging (iMRI), proposed by Bernays et al. in 2002 [4].

The iMRI guidance provides near real-time imaging during a neurosurgical procedure. It provides an image of the actual intraoperative situation, which can be useful when taking into account brain-shift. This phenomenon, almost always observed during extensive cranial procedures, can occur even during minor procedures such as STx biopsy $[24,25]$. Besides the obvious indications for the use of iMRI guidance provided in previous papers, including STx biopsy of very small, deeply localized or cystic lesions [4, 19,23], it is particularly useful in two other circumstances. The first is when a high resolution three-dimensional (3D) volume modality was not obtained during preoperative diagnostic imaging. High resolution 3D MR or CT images are used as reference examinations allowing the preoperative so called registration of the patient's head in the neuronavigation system. Points collected from the patient's head are subsequently merged with the surface of the patient's head 3D model developed basing on the previously mentioned 3D modalities. When using low-field iMRI, there is no need for head registration, which makes it unnecessary to perform additional preoperative imaging given the associated accumulative increase in cost and risks [3, 19, 22, 26, 27]. In our study, the 
total number of patients with insufficient neuroimaging - who were scheduled for the STx biopsy basing on a conventional MR study, which did not contain this particular sequence-is over $30 \%$.

Moreover, frameless iMRI-guided STx biopsy is relatively uncomplicated and technically straightforward and can be introduced as the first procedure during training in neurosurgical intraoperative imaging.

Although the usefulness of ultra-low-field iMRI in STx biopsy was subsequently confirmed by other authors, according to our knowledge, no previous published studies have compared iMRI to preoperative MRI for a brain tumour biopsy according to evidence-based medicine (EBM) guidelines [7, 19, 23]. Though this method has been subject to slight criticism, it has consequently been applied in neurosurgical daily practise in recent years $[1,25,29]$.

The aim of our study was to verify the safety and effectiveness of the STx biopsy guided by low-field iMRI in comparison with its frameless classic analogue basing on a prospective randomized parallel-group, controlled trial. In the current paper, we present the study design and results of the interim analysis.

\section{Material and methods}

\section{Patients}

Between June 2009 and August 2012, patients who were-follo wing contemporary recommendations [28] — scheduled to undergo STx biopsy were prospectively recruited for the study. The majority of the patients suffered from superficially localized tumours and was scheduled for an open surgery unless a radiologist suspected lymphoma or the general medical condition of the patient was unsatisfactory. An exception from this rule was made for pathologies localized in the eloquent brain area. In general, in such cases, we performed STx biopsy prior to the surgical excision to adjust the extent and technique of the desired subsequent resection basing on the obtained histopathology. An approval of the local ethics committee was obtained, and each patient signed a written consent to participate in the study. Graphical illustration of the recruitment and allocation process is presented in Fig. 1.

\section{Inclusion criteria}

The inclusion criteria were as follows: male and female patients $\geq 18$ years with supratentorial brain tumour scheduled to undergo STx biopsy. The estimated number of patients needed to reveal the difference of over $5 \%$ between primary endpoints'-diagnostic yield and complications ratio-at the level of significance 0.05 and power $80 \%$ was 465 per each arm $[32,34]$.

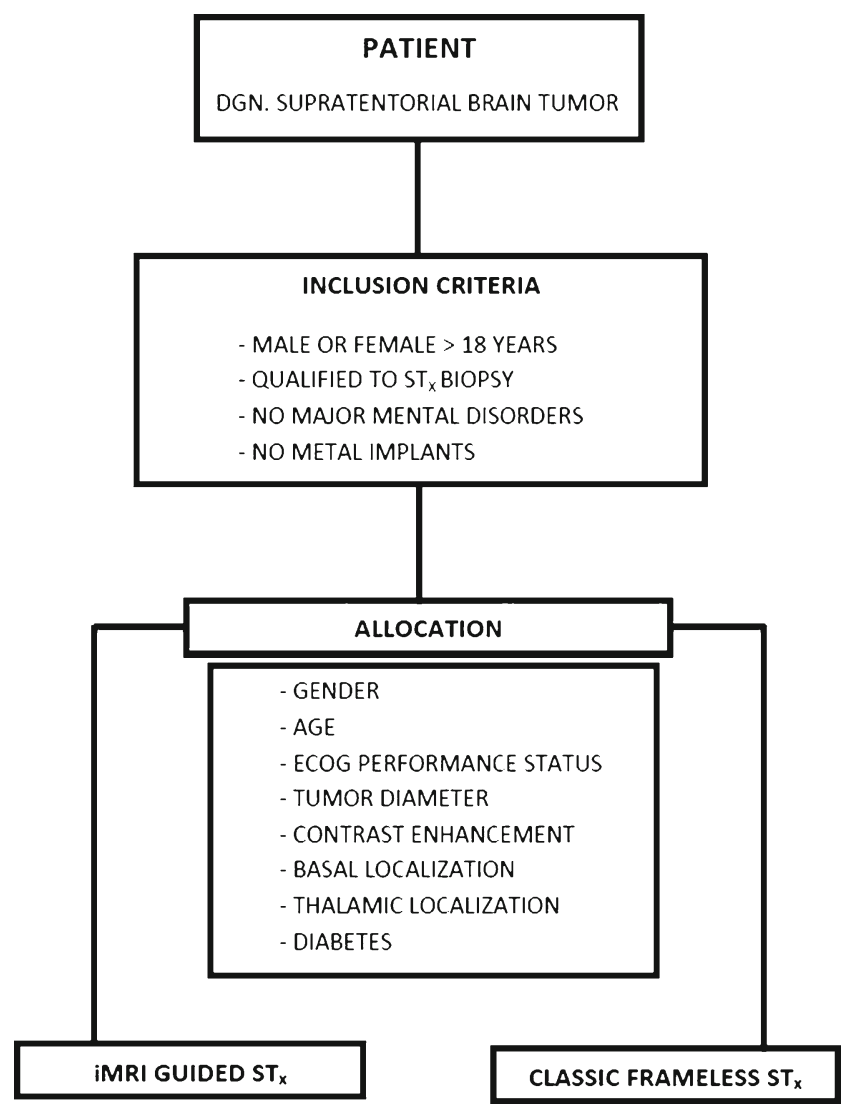

Fig. 1 Graphical illustration of the recruitment and randomization process. DGN diagnosed, STx biopsy stereotactic biopsy

\section{Exclusion criteria}

Patients unable to provide informed consent and those with metal implants which could disrupt or influence the head MR study were excluded from the study.

\section{Allocation}

Patients were prospectively allocated into the iMRI and the control group by minimization according to demographic (gender and age) and epidemiologic data (preoperative Eastern Cooperative Oncology Group (ECOG) performance status - a scale providing information about neurological and social status of a patient with oncological disease, maximum tumour diameter, presence of contrast enhancement, independent risk factors of haemorrhage — basal and thalamic localization and preoperative diabetes) $[10,15,17,18]$.

Intervention

After being transferred to the operating room, each patient was sedated with an intravenous infusion of remifentanil with passive oxygen therapy and monitoring of vital functions. Additionally, the sites of head holder pins and skin incision 
were anaesthetized with $1 \%$ lignocaine. All biopsies in both groups were performed via a 6-mm-burr hole with the use of the Vertec system (Medtronic Navigation, Louisville, CO, USA). A ferromagnetic passively navigated $2.2-\mathrm{mm}$ sidecut with a 9-mm-cutting window length biopsy needle was used (Fig. 2). Thus, each sample had an estimated volume of over $30 \mathrm{~mm}^{3}$. All operations were performed by one of the three first authors.

In the iMRI-guided group, the head of each patient was immobilized with a three-pin iMRI-compatible headholder. The PoleStar N20 iMRI system (Medtronic Navigation, Louisville, CO, USA) with a $0.15 \mathrm{~T}$ constant magnet was used in all procedures. Subsequently, after the patient's positioning, the preoperative reference examination was routinely carried out (T1+gadolinum, T2 or FLAIR-weighted - depending on the pathology and axial $4 \mathrm{~mm}$ scans). Images were automatically transferred into the neuronavigation system (StealthStation, Medtronic Navigation, Louisville, CO, USA). The entry point, target and optimal biopsy trajectory were then defined by the operator on the basis of the obtained iMRI images. Serial tissue samples (four from the central and another four from the marginal part of the tumour) were collected according to the modified protocol described by Shooman et al., which made the use of an intraoperative histopathological examination obsolete [28]. Following each operation, a control iMRI (T1weighted, axial and 4-mm scan examination) was routinely performed to confirm and document the proper targeting and to exclude postoperative hyperacute intraparenchymal bleeding as proposed by Bernays et al. $[4,7]$ (Fig. 3).

A frameless STx biopsy was performed for each patient from the control group with the use of a neuronavigation system. The entry point, target and optimal biopsy trajectory were defined by the operator before the procedure on the basis of the preoperatively obtained high-field MR images with the use of a neuronavigation workstation (Cranial 5; StealthStation Application Software, Medtronic Navigation, Louisville, CO, USA). A schematic presentation of the intervention in both the study and control groups is presented in Fig. 4.

Following surgery, the specimens were sent for independent histopathological analysis. An open resection was subsequently performed in four patients in the iMRI group and five in the non-iMRI group.

\section{Postoperative care}

Postoperative care was conducted according to standard protocols and clinical guidelines. A postoperative followup head CT was subsequently performed 4 to $6 \mathrm{~h}$ after each procedure. All patients were followed up with a clinical examination 2 weeks postoperatively performed by an blinded for the allocation investigator.

\section{Data collection}

Demographic and epidemiological data were collected prospectively. The primary endpoints were the ratio of acute postoperative complications and the diagnostic yield. The presence of acute postoperative complications was noted if any of the following findings were present: wound site infection up to 2 weeks after the operation, a new neurological deficit developed up to $24 \mathrm{~h}$ following the operation and present in a follow up clinical examination 2 weeks postoperatively and intraparenchymal hematoma with radiological or clinical signs of the intracranial expansion. The diagnostic yield was expressed according to literature as a percentage of patients in whom the histopathological diagnosis was possible on the basis of the biological material obtained during the operation [28]. Secondary endpoints included the preoperative $\left(\operatorname{LOS}_{\text {pre }}\right)$, postoperative $\left(\operatorname{LOS}_{\text {post }}\right)$ and total length of hospital stay (LOS) as well as the preparation $\left(\mathrm{T}_{\text {prep }}\right)$, operation $\left(\mathrm{T}_{\mathrm{op}}\right)$ and total operating room $\left(\mathrm{T}_{\mathrm{OR}}\right)$ time. The LOS and $\mathrm{T}$ were routinely measured and recorded in the central hospital files by independent staff.

\section{Statistical analysis}

The study and control group were compared statistically using STATISTICA v. 9.1 (StatSoft, Inc.) software. Binomial dichotomized data (gender, tumour enhancement ratio, independent bleeding risk factors and diagnostic yield) were compared with

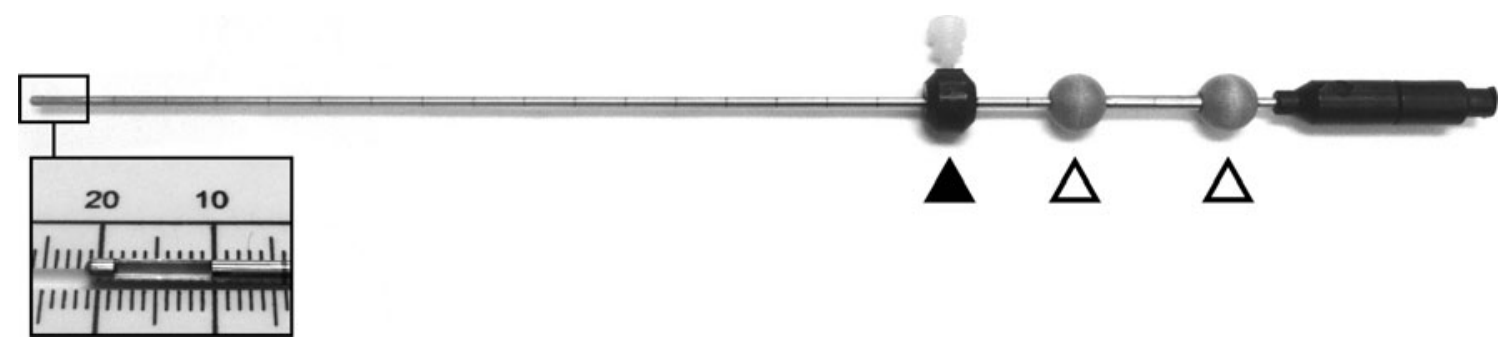

Fig. 2 A ferromagnetic passively navigated sidecut 2.2-mm biopsy needle. The solid triangle indicates a stopper, allowing to mark the depth of the needle insertion calculated by the neuronavigation system. Open triangles indicate passive neuronavigation markers. A close-up view of a 9-mm long cutting window of the biopsy needle allowing to obtain a tissue sample of the estimated volume of over $30 \mathrm{~mm}^{3}$ is visible in the lower-left corner 
Fig. 3 Example postoperative iMRI T1-weighted axial 4-mm control scans. a The left temporal lobe contrastenhancing tumour. b The left frontal lobe non-enhancing pathology. The ferromagnetic biopsy needle has been removed prior to scanning. The biopsy site is visible as a black spot-a small air bubble is indicated by the open triangles
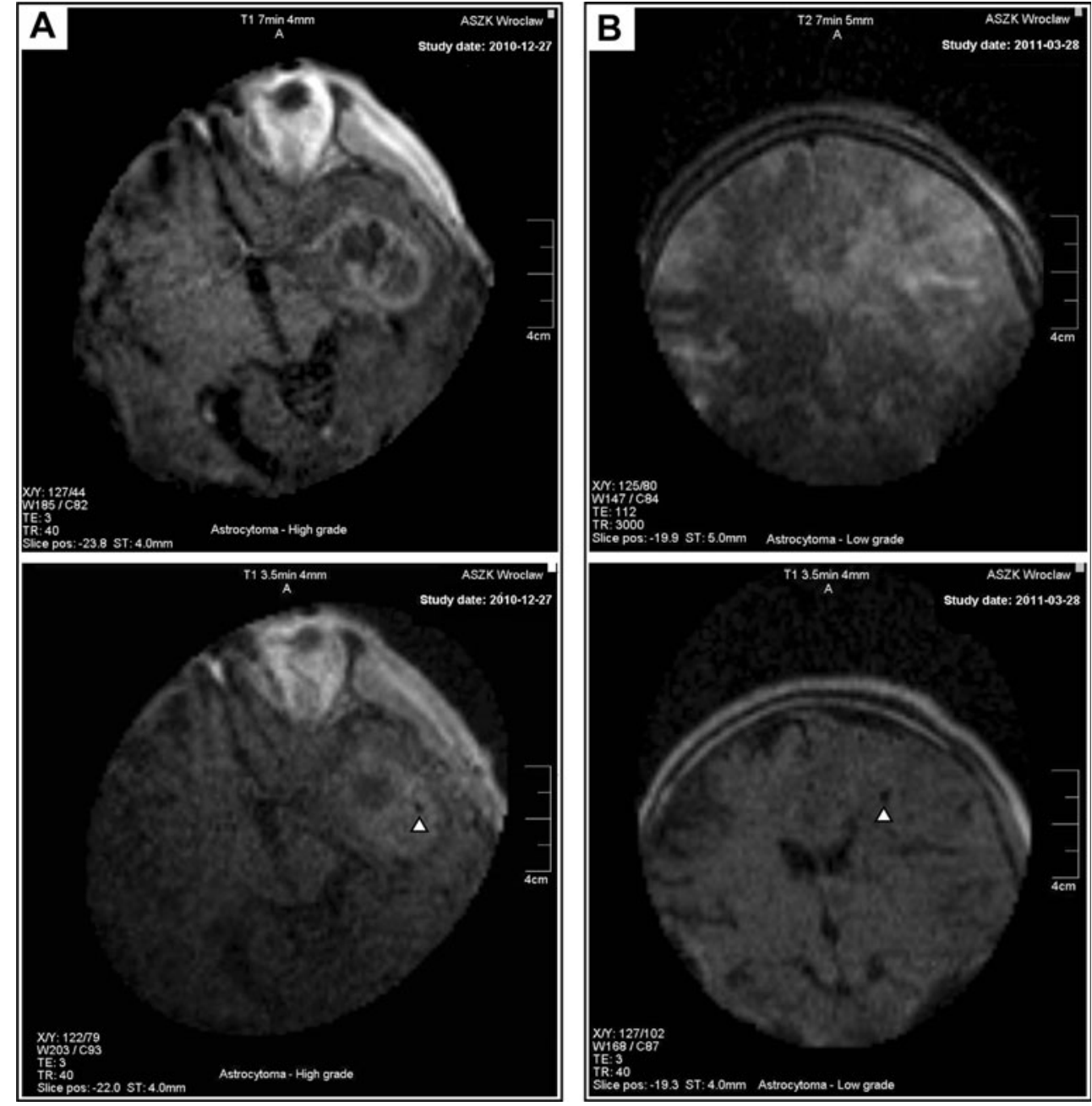

the two-tailed Fisher's exact test. After testing for normal distribution using the Shapiro-Wilk $W$ test, the Student's $t$ test was applied to compare patients' age, maximum tumour diameter and time of preparation and operation as well as total OR time. Differences between skewed data were compared: preoperative ECOG score and LOS with the Mann-Whitney $U$ test. A $p$ value of $\leq 0.05$ was considered statistically significant.

This study is registered with clinical trials. Gov. number NCT 01779219.

\section{Study termination criteria}

The interim analysis was performed each year of the study for safety reasons. We intended to terminate the study if a complication rate exceeded $6 \%$ (exceeding an average morbidity value reported in literature of 5.5 by $10 \%$ ) or if the revealed diagnostic yield would be lower than $88 \%$ (5\% less than the average $93 \%$ value of the diagnostic yield reported in literature) in either group [4, 13, 17, 20, 28, 30]. Similarly to previous interim analyses, we did not reveal such violations of the contemporary standards; therefore, the study was continued. A considered positive reason for the premature termination of the study would be the lack of significant differences in terms of primary endpoints revealed during the interim analysis of groups exceeding 100 cases each.

\section{Results}

The inclusion criteria were met by 42 patients, who were enrolled in the study as a pilot group. Both the iMRI and control group consisted of 21 patients. The iMRI and the control group did not differ significantly in relation to: gender $(p=0.75)$, age $(p=0.45)$, preoperative ECOG performance status $(p=0.86)$, maximum tumour diameter $(p=0.42)$, enhancement ratio $(p=0.50)$ and independent risk factors for haemorrhage - basal $(p=1.00)$ and thalamic $(p=0.66)$ localization of the pathology and preoperative diabetes $(p=1.00)$. Essential demographic and epidemiologic data are presented in Tables 1 and 2.

The obtained biopsies were suitable for neuropathological studies in 20 (95\%) cases from both the iMRI and control 
Fig. 4 Schematic presentation of the intervention in both study and control group. STx biopsy stereotactic biopsy, post-op postoperative, $e$-steady iMRI scan modality routinely performed during preoperative positioning of the patient, $a x$ axial scan orientation, $\min$ minutes, $s$ seconds

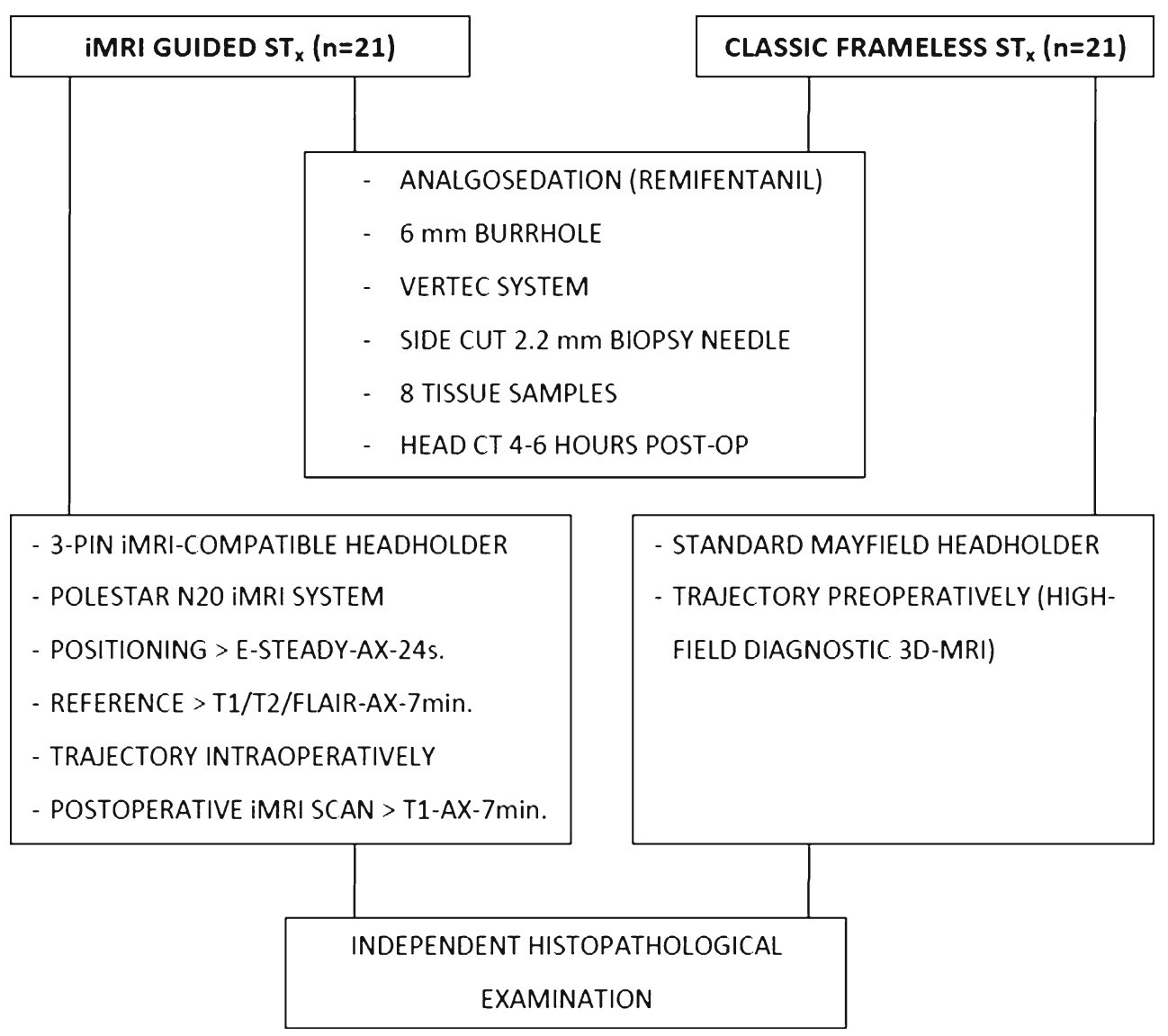

group ( $p=1.00)$. In terms of postoperative complications, one case of intraparenchymal haemorrhage, treated conservatively, was noted in the control group $(p=1.00)$. We did not reveal any cases of hyperacute bleeding in the postoperative iMRI scans.

The total, pre- and postoperative lengths of hospital stay were not statistically significantly different between both groups. The mean $\mathrm{T}_{\text {prep }}$ and $\mathrm{T}_{\mathrm{op}}$ differed between both groups ( $p=0.004$ and $p=0.024$, respectively). The mean $\mathrm{T}_{\mathrm{OR}}$ was $111 \pm 24 \mathrm{~min}$ (iMRI) and $78 \pm 29 \mathrm{~min}$ (control) and also differed significantly $(p<0.0001)$. The summary of dependent variables supplemented by results of the statistical analysis is presented in Table 3 and Fig. 5.

\section{Discussion}

The use of iMRI guidance for STx biopsies allows to overcome the lack of a preoperative high-field 3D study and to perform the procedure basing on near real-time imaging. In the interim analysis of our prospective, randomized and controlled trial, we noted that both types of biopsies - with and without intraoperative imaging - were comparably effective and safe with the prolongation of the iMRI-guided procedure over $30 \mathrm{~min}$ as compared to the classically navigated one.

In 2002, Bernays et al. stated that intraoperative imaging with the use of low-field intraoperative magnetic resonance may improve diagnostic yield - mainly through the avoidance of the brain-shift phenomenon influence - with relatively low morbidity and low complication rates [4]. Since then, a number of authors confirmed these findings based on relatively small cohort observations [7, 19]. Additionally, as proposed by Schulder and Spiro, iMRI makes intraoperative histopathology obsolete and can be routinely used for stereotactic biopsies in a regular neurosurgical practise [23]. However, it should also be taken into account that the use of iMRI may prolong and technically complicate the operation [9, 24, 25]. Additionally, the quality of the intraoperatively obtained 4-mm-thick scans, compared to the high-field (1.5 or $3 \mathrm{~T}$ ) 3D volume diagnostic ones, may be considered insufficient to plan the optimal biopsy trajectory in relation to all the critical structures such as cortical or peritumoural vessels. These may — at least theoretically_ provide an increased risk of morbidity or misdiagnosis.

Due to the fact that the main objective of the implementation of iMRI is usually real intraoperative imaging, one may not expect that it could also reasonably replace preoperative high resolution modalities, which is the major case in our study. The true value of iMRI guidance in STx biopsies has not been validated scientifically. Until now, there are no papers available comparing iMRI to preoperative MRI for STx biopsies. Therefore, there is an unquestionable need to verify the effectiveness and safety of the use of iMRI to conduct frameless brain tumour biopsies, preferably using the principles of $\operatorname{EBM}[12,21,33]$. 
Table 1 Characteristics of the patients. Completed with the allocation information, demographic and epidemiological information as well as pathology description

\begin{tabular}{|c|c|c|c|c|c|c|c|c|c|}
\hline & Allocation & Sex & Age & WHO & Maximum Diameter [mm] & Enhancement & Dominant hemisphere & Lobe & Pathology \\
\hline P 01 & Non-iMRI & Male & 36 & 3 & 26 & - & - & $\mathrm{F}+\mathrm{P}$ & Limph \\
\hline P 02 & iMRI & Female & 77 & 2 & 52 & + & - & $\mathrm{T}+\mathrm{P}$ & Hgg \\
\hline P 03 & Non-iMRI & Female & 67 & 2 & 45 & + & + & $\mathrm{T}+\mathrm{P}$ & Hgg \\
\hline P 04 & iMRI & Female & 60 & 2 & 55 & + & - & $\mathrm{P}$ & Hgg \\
\hline P 05 & iMRI & Male & 46 & 2 & 69 & + & + & $\mathrm{T}$ & Hgg \\
\hline P 06 & iMRI & Female & 64 & 1 & 25 & + & + & $\mathrm{P}$ & Infl \\
\hline P 07 & Non-iMRI & Male & 57 & 1 & 60 & + & + & $\mathrm{T}+\mathrm{P}$ & Limph \\
\hline P 08 & Non-iMRI & Male & 56 & 2 & 29 & - & - & $\mathrm{F}$ & - \\
\hline Р 09 & Non-iMRI & Female & 40 & 2 & 31 & - & + & $\mathrm{F}$ & Lgg \\
\hline P 10 & iMRI & Female & 77 & 3 & 69 & + & - & $\mathrm{P}$ & Hgg \\
\hline P 11 & iMRI & Female & 48 & 2 & 64 & + & + & $\mathrm{F}$ & Hgg \\
\hline P 12 & Non-iMRI & male & 47 & 2 & 64 & + & - & $\mathrm{F}$ & Hgg \\
\hline P 13 & Non-iMRI & Female & 73 & 2 & 50 & + & + & $\mathrm{P}$ & Hgg \\
\hline P 14 & Non-iMRI & Female & 65 & 3 & 77 & - & + & $\mathrm{T}+\mathrm{P}$ & Lgg \\
\hline P 15 & iMRI & Male & 73 & 3 & 39 & + & - & $\mathrm{T}$ & Limph \\
\hline P 16 & iMRI & Male & 22 & 1 & 36 & - & + & $\mathrm{F}$ & Lgg \\
\hline P 17 & Non-iMRI & Male & 61 & 2 & 45 & + & + & $\mathrm{F}$ & Hgg \\
\hline P 18 & Non-iMRI & Male & 80 & 3 & 38 & - & - & $\mathrm{T}$ & Lgg \\
\hline P 19 & iMRI & Male & 19 & 1 & 8 & + & - & $\mathrm{T}$ & Limph \\
\hline P 20 & Non-iMRI & Male & 60 & 1 & 50 & + & + & $F+T+P$ & Hgg \\
\hline P 21 & Non-iMRI & Female & 77 & 3 & 42 & + & - & $\mathrm{T}$ & Hgg \\
\hline P 22 & iMRI & Male & 44 & 1 & 60 & - & + & $\mathrm{F}+\mathrm{T}$ & Lgg \\
\hline P 23 & iMRI & Female & 46 & 3 & 24 & + & - & $\mathrm{P}$ & Limph \\
\hline P 24 & iMRI & Male & 34 & 2 & 64 & + & + & $\mathrm{T}$ & Lgg \\
\hline P 25 & iMRI & Male & 50 & 3 & 59 & + & - & $\mathrm{P}+\mathrm{O}$ & Limph \\
\hline P 26 & iMRI & Male & 64 & 2 & 60 & + & - & $\mathrm{F}$ & Hgg \\
\hline P 27 & iMRI & Male & 63 & 2 & 35 & - & + & $\mathrm{F}+\mathrm{T}$ & Lgg \\
\hline P 28 & Non-iMRI & Male & 51 & 1 & 51 & + & + & $\mathrm{T}$ & Hgg \\
\hline P 29 & Non-iMRI & Male & 38 & 0 & 90 & - & - & $\mathrm{F}+\mathrm{T}+\mathrm{P}$ & Lgg \\
\hline P 30 & Non-iMRI & Male & 52 & 4 & 67 & + & - & $\mathrm{F}$ & Hgg \\
\hline P 31 & iMRI & Male & 57 & 3 & 46 & + & - & $\mathrm{F}$ & Limph \\
\hline P 32 & Non-iMRI & Male & 60 & 2 & 48 & + & - & $\mathrm{T}$ & Hgg \\
\hline P 33 & Non-iMRI & Female & 71 & 2 & 52 & + & - & $\mathrm{T}$ & Hgg \\
\hline P 34 & iMRI & Male & 50 & 1 & 74 & - & + & $F+P$ & Lgg \\
\hline P 35 & Non-iMRI & Male & 49 & 2 & 48 & + & + & $\mathrm{P}+\mathrm{O}$ & Hgg \\
\hline P 36 & Non-iMRI & Female & 32 & 0 & 71 & - & + & $\mathrm{F}$ & Lgg \\
\hline P 37 & iMRI & Male & 32 & 1 & 16 & - & + & $\mathrm{F}$ & Infl \\
\hline P 38 & iMRI & Female & 28 & 1 & 66 & + & + & $\mathrm{T}$ & Hgg \\
\hline P 39 & Non-iMRI & Female & 63 & 2 & 80 & + & - & $\mathrm{T}+\mathrm{O}$ & Hgg \\
\hline P 40 & iMRI & Male & 72 & 3 & 62 & + & + & $\mathrm{F}$ & - \\
\hline P 41 & Non-iMRI & Female & 29 & 0 & 75 & - & - & $\mathrm{F}+\mathrm{T}$ & Lgg \\
\hline P 42 & iMRI & Male & 60 & 2 & 35 & + & - & $\mathrm{T}$ & Lgg \\
\hline
\end{tabular}

$F$ frontal lobe, $P$ parietal lobe, $T$ temporal lobe, $O$ occipital lobe, $L g g$ low grade gliomas, $H g g$ high grade gliomas, Limph liphoma, Infl inflammation

Our study is a prospective randomized trial, which is its obvious strength. It is open-label, with no blinding of patients and investigators and with no attempt made at allocation concealment which could be considered as a pitfall. These features of the study were necessitated by the specifics of the intervention [25]. Additionally, the histopathological assessment 
Table 2 Independent variables analysed among iMRI and control group. Mean \pm standard deviation, median with (interquartile range) or percentile values were expressed

\begin{tabular}{llll}
\hline Variables & iMRI $(n=21)$ & Control $(n=21)$ & $P$ \\
\hline Age & $52 \pm 17$ & $55 \pm 15$ & 0.46 \\
Gender [male] & $67 \%$ & $57 \%$ & 0.75 \\
ECOG performance status $^{\mathrm{a}}$ & $2(2)$ & $2(1)$ & 0.86 \\
Maximum tumour diameter [mm] $^{\text {Tumour enhancement ratio }}$ & $48 \pm 19$ & $54 \pm 18$ & 0.32 \\
Bleeding risk factors & $76 \%$ & $62 \%$ & 0.50 \\
Basal localization & & & 1.00 \\
Thalamic localization & $38 \%$ & $43 \%$ & 0.66 \\
Diabetes & $10 \%$ & $19 \%$ & 1.00 \\
\hline
\end{tabular}

${ }^{a}$ Eastern Cooperative Oncology Group performance status - a scale which provides information about neurological and social status and independency of patient with oncological disease

${ }^{\mathrm{b}}$ According to McGirt et al. [17]

was done by an independent specialist who was masked to the treatment allocation.

An important point of the study is the fact that it was not possible to compare results of the histopathological examination of the biopsy specimens with those of the examination of all of the tumour tissue. This was due to a small number of patients who underwent subsequent tumour resection. As we obtained relatively large tissue samples (each sample volume reaching $30 \mathrm{~mm}^{3}$ ), we decided to analyse the diagnostic yield, expressing the number of cases in which it was possible to obtain reliable results of histopathological examination. The same methodology was implemented by numerous previous authors assessing or comparing various techniques of stereotactic biopsy $[4,8,13,28]$. Our findings might also be restricted by the fact that our study was conducted at only one centre. However, three surgeons performed operations, which may provide generalizability.

Our results were comparable with those from available literature in terms of the diagnostic yield: $95 \%$ in both groups versus a reported average of $93 \%$ (between 92 and $100 \%$ ) and safety - no observed significant complications in both groups versus an average of $5.5 \%$ incidence of morbidity reported in literature $[4,13,17,20,28,30]$. We did not reveal significant difference in terms of the length of hospital stay between both groups. An insignificant shortening of total LOS in the iMRI arm may be caused by the lack of need for preoperative imaging dedicated to neuronavigation purposes. The number of patients admitted without full

Table 3 The summary of dependent variables supplemented by results of the statistical analysis. Mean \pm standard deviation, median with (interquartile range) or percentile values were expressed

\begin{tabular}{llll}
\hline Variables & iMRI $(n=21)$ & Control $(n=21)$ & \\
\hline Time [min] & & & \\
$\mathrm{T}_{\text {prep }}$ & $53 \pm 22$ & $34 \pm 20$ & 0.004 \\
$\mathrm{~T}_{\text {op }}$ & $60 \pm 26$ & $74 \pm 20$ & 0.024 \\
$\mathrm{~T}_{\text {OR }}$ & $111 \pm 24$ & & \\
Complications & & 1 & \\
Haematoma & 0 & 0 & \\
Neurological deterioration & 0 & 0 & \\
Infection & 0 & $95 \%$ & \\
Diagnostic yield & $95 \%$ & & - \\
Length of hospital stay [days] & & $4(4)$ & \\
LOS $_{\text {pre }}$ & $3(4)$ & $2(3)$ & \\
LOS $_{\text {post }}$ & $2(4)$ & $7(3)$ & 0.001 \\
LOS $_{\text {TOTAL }}$ & $5(5)$ & 1.00 \\
\hline
\end{tabular}

$\mathrm{T}_{\text {prep }}$ - time of the preparation (period from the moment of conscious patient arriving to the operating room to the moment of skin incision), $\mathrm{T}_{\mathrm{op}}$ - time of the operation (period from the moment of skin incision to last suture insertion), $\mathrm{T}_{\mathrm{OR}}$ - total or time $\left(\mathrm{T}_{\mathrm{prep}}+\mathrm{T}_{\mathrm{op}}\right)$. $\mathrm{LOS}_{\mathrm{pre}}-$ preoperative length of hospital stay, $\operatorname{LOS}_{\text {post }}$ - postoperative length of hospital stay, $\operatorname{LOS}_{\mathrm{TOTAL}}$ - total length of hospital stay 
Fig. 5 Box graphs showing the differences between groups in a mean total length of hospital stay and $\mathbf{b}$ median total OR time. $P$ values for MannWhitney $U($ a) and Student's $t$ test (b) are presented
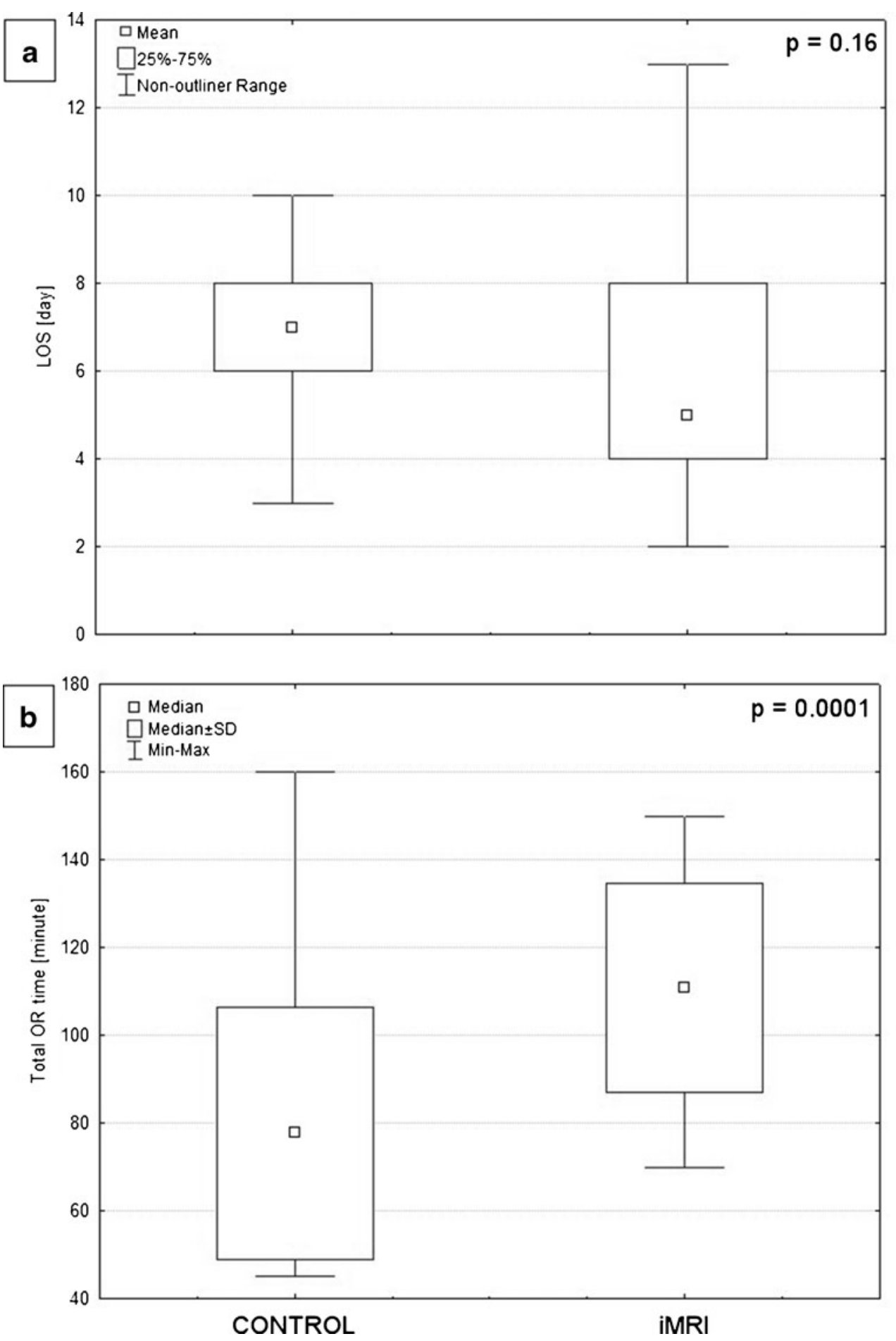

radiological diagnostic testing in our study was 30\%-which gives an average of seven patients in each group. There is an undeniable need to verify these theses basing on a wider group of participants.

In our trial, the iMRI guidance added approximately $30 \mathrm{~min}$ to conventional surgery time. Pre- and potential intraoperative image acquisition could usually be done within a few minutes, but preparation of the operating room and positioning of the patient in the MRI-compatible head holder are more difficult than placing the patient's head in a regular head fixation (e.g., a Mayfield clamp). However, total time demands could be different if the time spent by the operator on preoperative planning would be included in the total OR time in the non-iMRI arm.

The independent variables were chosen with a special emphasis on making the groups comparable demographically as well as in terms of the risk factors for bleeding, neurological 
complications or infection [17]. As neither the tumour localization nor the biopsy trajectory length or its transition through the brain eloquent areas were recognized as independent risk factors for the postoperative complications, we decided not to take them into account as factors influencing the allocation $[17,20]$. We chose minimization as a method of allocation which could also be considered as a limitation of our study. Treatment allocation by minimization - in which the first study participant is allocated to a treatment arm at random and all subsequent participants are allocated based on which treatment arm assignment would result in a better balance between the study groups relative to the variable of interest - is not the same as randomization [10]. However, since the desired number of participants required to reveal the difference of over $5 \%$ between parameters was estimated at the level of 465 patients per each group, we decided to make both the study and control group comparable in terms of independent variables at each step of the experiment [6,31,34].

Based on available literature stating that non-ferromagnetic biopsy needles may provide tissue samples of lower histopathological quality, we decided to use a standard side cutting of $2.2 \mathrm{~mm}$ passively navigated ferromagnetic needles in both groups [16]. This made proper intraoperative visualization of the needle tip impossible. Although we discovered the presence of a small air bubble in the site of tissue sampling in all cases, at the moment, we do not feel authorized to relay on this promising finding previously described by Czyz et al. [7]. The number of patients enrolled in the study so far is obviously insufficient to validate the usefulness of this supplementary method, but our study is ongoing and we look forward to further results. This may be treated as an inherent limitation of our study; since in all previous papers, authors have used iMRI to verify the accuracy of the biopsy needle placement $[4,19,23]$. One needs to notice that our intention was to assume the situation when the usage of iMRI guidance to plan and perform STx biopsy is favoured because of organizational conditions rather than obvious indications - biopsy of a cystic or deep-seated lesion $[4,23]$. If intraoperative visualization of the needle tip would be possible, one could expect an even higher diagnostic yield than be reported for STx biopsies carried out without iMRI. The general idea was to widen the indications for iMRI guidance in STx biopsy beyond the existing ones.

The possibility to detect a hyperacute intraparenchymal brain haematoma even with low-field MR imaging was suggested by numerous authors $[2,4,5,11,35]$. On the other hand, Küker et al. stated that the detection of hyperacute intraparenchymal blood requires the application of at least three MR sequences and is, therefore, complicated and time-consuming [14]. None of the patients from our iMRI-guided group suffered from postoperative intracerebral bleeding, thus, we did not have a chance to assess the validity of postoperative T1-weighted iMRI images in the detection or prediction of early haemorrhagic complications. Another noteworthy fact to the trial was that the studied tumours were all large (mean maximum diameters of 48 and $46 \mathrm{~mm}$ for the two groups). Given that low-resolution $(0.15 \mathrm{~T}) \mathrm{iMRI}$ is able to detect larger lesions better than the small ones, the qualification of the findings based on tumour size seems to be justified. Both aspects should be clearly assessed based on wider clinical material in the nearest future.

Because of the small number of patients involved in our study, one should consider our results with caution. At this point of our study we can conclude that the use of iMRI in stereotactic brain tumour procedures prolongs the time of the procedure but may be comparable in terms of safety and efficacy to the standard frameless stereotactic biopsy method. An obvious advantage of iMRI-guided biopsies is that it can be considered in cases where no preoperative 3D-MR scans were acquired, and in such a situation, this technique may prove time- and cost-effective.

Acknowledgments The authors are grateful to Dr. Dariusz Szarek from the Department of Neurosurgery, Wroclaw University Hospital for technical support in the study.

Open Access This article is distributed under the terms of the Creative Commons Attribution License which permits any use, distribution, and reproduction in any medium, provided the original author(s) and the source are credited.

\section{References}

1. Arle JE (2011) Evidence-based medicine: fact or fiction? World Neurosurg 76:45-47

2. Atlas SW, Thulborn KR (1998) MR detection of hyperacute parenchymal hemorrhage of the brain. AJNR Am J Neuroradiol 19:1471-1477

3. Baumann F, Schmid C, Bernays RL (2010) Intraoperative magnetic resonance imaging-guided transsphenoidal surgery for giant pituitary adenomas. Neurosurg Rev 33:83-90

4. Bernays RL, Kollias SS, Khan N, Brandner S, Meier S, Yonekawa Y (2002) Histological yield, complications, and technological considerations in 114 consecutive frameless stereotactic biopsy procedures aided by open intraoperative magnetic resonance imaging. J Neurosurg 97:354-362

5. Bradley WG Jr (1993) MR appearance of hemorrhage in the brain. Radiology 189:15-26

6. Charles P, Giraudeau B, Dechartres A, Baron G, Ravaud P (2009) Reporting of sample size calculation in randomised controlled trials: review. BMJ 338:b1732

7. Czyz M, Tabakow P, Jarmundowicz W, Lechowicz-Glogowska B (2012) Intraoperative magnetic resonance - guided frameless stereotactic biopsies - initial clinical experience. Neurol Neurochir Pol 46:157-160

8. Frati A, Pichierri A, Bastianello S, Raco A, Santoro A, Esposito V, Giangaspero F, Salvati M (2011) Frameless stereotactic cerebral biopsy: our experience in 296 cases. Stereotact Funct Neurosurg 89:234-245

9. Gerlach R, du Mesnil de Rochemont R, Gasser T, Marquardt G, Reusch J, Imoehl L, Seifert V (2008) Feasibility of PoleStar N20, an ultra-low-field intraoperative magnetic resonance imaging system in resection control of pituitary macroadenomas: lessons 
learned from the first 40 cases. Neurosurgery 63:272-284, discussion 284-275

10. Han B, Enas NH, McEntegart D (2009) Randomization by minimization for unbalanced treatment allocation. Stat Med 28:3329-3346

11. Hayman LA, McArdle CB, Taber KH, Saleem A, Baskin D, Lee HS, Kirkpatrick JB, Herrick RC, Bryan RN (1989) MR imaging of hyperacute intracranial hemorrhage in the cat. AJNR Am J Neuroradiol 10:681-686

12. Isaacs D, Fitzgerald D (1999) Seven alternatives to evidence-based medicine. BMJ 319:1618

13. Jain D, Sharma MC, Sarkar C, Gupta D, Singh M, Mahapatra AK (2006) Comparative analysis of diagnostic accuracy of different brain biopsy procedures. Neurol India 54:394-398

14. Kuker W, Thiex R, Rohde I, Rohde V, Thron A (2000) Experimental acute intracerebral hemorrhage. Value of MR sequences for a safe diagnosis at 1.5 and 0.5 T. Acta Radiol 41:544-552

15. Kundt G (2009) Comparative evaluation of balancing properties of stratified randomization procedures. Methods Inf Med 48:129-134

16. Langen HJ, Kugel H, Ortmann M, Noack M, de Rochemont RM, Landwehr P (2001) Functional capacity of MRI-compatible biopsy needles in comparison with ferromagnetic biopsy needles. In vitro studies. Rofo 173:658-662

17. McGirt MJ, Woodworth GF, Coon AL, Frazier JM, Amundson E, Garonzik I, Olivi A, Weingart JD (2005) Independent predictors of morbidity after image-guided stereotactic brain biopsy: a risk assessment of 270 cases. J Neurosurg 102:897-901

18. Oken MM, Creech RH, Tormey DC, Horton J, Davis TE, McFadden ET, Carbone PP (1982) Toxicity and response criteria of the Eastern Cooperative Oncology Group. Am J Clin Oncol 5:649-655

19. Quinn J, Spiro D, Schulder M (2011) Stereotactic brain biopsy with a low-field intraoperative magnetic resonance imager. Neurosurgery 68:217-224, discussion 224

20. Sawin PD, Hitchon PW, Follett KA, Torner JC (1998) Computed imaging-assisted stereotactic brain biopsy: a risk analysis of 225 consecutive cases. Surg Neurol 49:640-649

21. Scholler K, Licht S, Tonn JC, Uhl E (2009) Randomized controlled trials in neurosurgery - how good are we? Acta Neurochir (Wien) 151:519-527, discussion 527

22. Schulder M, Carmel PW (2003) Intraoperative magnetic resonance imaging: impact on brain tumor surgery. Cancer Control 10:115-124

23. Schulder M, Spiro D (2011) Intraoperative MRI for stereotactic biopsy. Acta Neurochir Suppl 109:81-87

24. Seifert V, Gasser T, Senft C (2011) Low-field intraoperative MRI in glioma surgery. Acta Neurochir Suppl 109:35-41

25. Senft C, Bink A, Franz K, Vatter H, Gasser T, Seifert V (2011) Intraoperative MRI guidance and extent of resection in glioma surgery: a randomised, controlled trial. Lancet Oncol 12:9971003

26. Senft C, Franz K, Ulrich CT, Bink A, Szelenyi A, Gasser T, Seifert V (2010) Low-field intraoperative MRI-guided surgery of gliomas: a single center experience. Clin Neurol Neurosurg 112:237-243

27. Senft C, Seifert V, Hermann E, Gasser T (2009) Surgical treatment of cerebral abscess with the use of a mobile ultralow-field MRI. Neurosurg Rev 32:77-84, discussion 84-75

28. Shooman D, Belli A, Grundy PL (2010) Image-guided frameless stereotactic biopsy without intraoperative neuropathological examination. J Neurosurg 113:170-178

29. Stummer W, Nestler U, Stockhammer F, Krex D, Kern BC, Mehdorn HM, Vince GH, Pichlmeier U (2011) Favorable outcome in the elderly cohort treated by concomitant temozolomide radiochemotherapy in a multicentric phase II safety study of 5-ALA. J Neurooncol 103:361-370

30. Teixeira MJ, Fonoff ET, Mandel M, Alves HL, Rosemberg S (2009) Stereotactic biopsies of brain lesions. Arq Neuropsiquiatr 67:74-77
31. Weaver CS, Leonardi-Bee J, Bath-Hextall FJ, Bath PM (2004) Sample size calculations in acute stroke trials: a systematic review of their reporting, characteristics, and relationship with outcome. Stroke 35:1216-1224

32. Wittes J (2002) Sample size calculations for randomized controlled trials. Epidemiol Rev 24:39-53

33. Yarascavitch BA, Chuback JE, Almenawer SA, Reddy K, Bhandari M (2012) Levels of evidence in the neurosurgical literature: more tribulations than trials. Neurosurgery Rev 6:1131-1137, discussion $1137-1138$

34. Zhong B (2009) How to calculate sample size in randomized controlled trial? J Thorac Dis 1:51-54

35. Zyed A, Hayman LA, Bryan RN (1991) MR imaging of intracerebral blood: diversity in the temporal pattern at 0.5 and $1.0 \mathrm{~T}$. AJNR Am J Neuroradiol 12:469-474

\section{Comments}

\section{Dieter Hellwig, Hanover, Germany}

This is a very interesting paper about frameless stereotactic biopsies using intraoperative low-field MRI guidance. Over years, the golden standard was the frame-based stereotactic technique to perform biopsies in histopathological unclarified space occupying brain lesions. The main drawbacks of the frame-based stereotactic technique were the lack of mobility and flexibility of the instrumentation as well as the so-called "blind" procedure. Therefore, the endoscope-assisted stereotactic biopsy has been introduced (1). In the last years, the combination of neuronavigation together with neuroendoscopy gains in importance, especially for biopsies of intraventricular tumours $(2,3)$. The main advantage is that the procedure is done under direct vision, and the surgeon has much more freedom in surgical planning and performance. In this study, Czyz and colleagues showed another suitable technique for "stereotactic" brain tumour biopsies in a safe and effective way. They used intraoperative low-field MRI as a guidance. The results seem to be comparable with other stereotactic bioptic techniques under special consideration of postoperative haematoma and the reliability of the histopathological diagnosis. In my opinion, the main drawback of this technique is that the authors used ferromagnetic needles, which make the intraoperative visualization of the needle tip impossible and jeopardize the tremendous advantage with intraoperative real time control of the biopsy forceps using iMRI.

\section{References}

1. Mennel H.D., Hellwig D, Bauer B.L. (1994) Results and reliability of brain tumour biopsies gained by stereotactic and endoscopic techniques. Zentralblatt Neurochir 55: 79-90

2. Tirakotai W, RIegel T, Sure U, Bozinov O, Hellwig D, Bertalanffy $\mathrm{H}$ (2004) Clinical application of neuro-navigation in a series of single burr-hole procedures. Zentralblatt Neurochir 65(2): 57-64

3. Constantini S, Mohanty A, Zymberg S, Cavalheiro S, Malluci C, Hellwig D, Ersahin Y, Mori H, Mascari C, Wagner W, Kulkarni AV, Sgouros S, Oi S (2013) Safety and diagnostic accuracy of neuroendoscopic biopsies: an international multicenter study. J Neurosurg Pediatr. (Epub ahead of print).

\section{Christian Senft, Frankfurt, Germany}

Here, Czyz and colleagues report the design of a randomized clinical trial concerning the use of a mobile intraoperative MRI in stereotactic biopsies, and they present data from an interim analysis after inclusion of a pilot group of 42 patients. First of all, the authors are to be congratulated to perform such a clinical trial. There is a growing need for solid clinical data to ground decision-making in surgery in general, particularly in neurosurgery. Especially the use of high-cost technology 
needs to be justified, and only data derived from randomized trials will convince health care providers to invest in this field. Intraoperative MRI was introduced into the field of neurosurgery to serve as a means of resection control in brain tumour surgery and has recently been proven to be beneficial in a randomized controlled trial [2]. Another area of use for this technology has been the field of minimal-invasive stereotactic procedures [1,3], yet without such high-class scientific proof: once completed, the study from Czyz and colleagues might be suitable to tell us neurosurgeons whether there is a benefit from using intraoperative MRI for these procedures as well. However, a large number of patients (>900) need to be enrolled according to the study design. I hope the authors will succeed with patient accrual and will not have to terminate their study early.
References

1. Bernays RL, Kollias SS, Khan N, Brandner S, Meier S, Yonekawa Y (2002) Histological yield, complications, and technological considerations in 114 consecutive frameless stereotactic biopsy procedures aided by open intraoperative magnetic resonance imaging. J Neurosurg 97 (2): 354-362

2. Senft C, Bink A, Franz K, Vatter H, Gasser T, Seifert V (2011) Intraoperative MRI guidance and extent of resection in glioma surgery: a randomised, controlled trial. The Lancet Oncology 12 (11): 9971003. doi:10.1016/S1470-2045(11)70196-6

3. Senft C, Seifert V, Hermann E, Gasser T (2009) Surgical treatment of cerebral abscess with the use of a mobile ultralow-field MRI. Neurosurg Rev 32 (1): 77-84; discussion 84-75. doi:10.1007/s10143-008-0174-0 